American Journal of Environmental Sciences 4 (5): 462-466, 2008

ISSN 1553-345X

(C) 2008 Science Publications

\title{
Potential Photooxidation Pathways of Dicarboxylic Acids in Atmospheric Droplets
}

\author{
${ }^{1}$ L.M. Yang, ${ }^{1}$ L.E. Yu and ${ }^{2}$ M.B. Ray \\ ${ }^{1}$ Department of Chemical \& Biomolecular Engineering and Environmental Science \& Engineering, \\ National University of Singapore, Singapore 119260 \\ ${ }^{2}$ Department of Chemical Engineering, University of Western Ontario, London \\ Ontario, N6A 5B9, Canada
}

\begin{abstract}
Low molecular weight (LMW) dicarboxylic acids (DCAs) have received increasing attention because of their hygroscopic properties and capability of acting as cloud condensation nuclei $(\mathrm{CCN})$. Based on the concentration profiles of intermediates identified during laboratory photooxidation of $\mathrm{C}_{7}-\mathrm{C}_{9}$ DCAs in a liquid-phase reaction system, chemometric analyses were conducted to examine the degradation pathways of DCAs for the formation of secondary DCAs. Among all secondary DCAs formed during the reaction, $\mathrm{C}_{4}$ and $\mathrm{C}_{5}$ DCAs showed the largest stoichiometric coefficients, indicating that sequential decarboxylation suggested in literature is unlikely the dominant pathway; other reaction routes e.g. preferential abstraction of hydrogen from carbon located at the central position of the DCA molecules by $\mathrm{OH}$, followed by subsequent decomposition through the breakage of $\mathrm{C}-\mathrm{C}$ bond, could play an important role.
\end{abstract}

Key words: Dicarboxylic acid, reaction pathway, intermediates, photooxidation

\section{INTRODUCTION}

Water-soluble organic species (WSOS) have recently received more attention because they have been assumed to be partially responsible for the water uptake of airborne particulates. Low molecular weight dicarboxylic acids (DCAs, $\mathrm{C}_{2}-\mathrm{C}_{9}$ DCAs), one of major classes of WSOS, contribute up to $50 \%$ of the organic aerosol mass ${ }^{[1]}$, can play an important role in both direct and indirect aerosol forcing ${ }^{[2,3,4,5]}$. Researchers have indicated that secondary formation through atmospheric oxidation could contribute to airborne DCAs ${ }^{[6,7,8]}$, while limited effort has been devoted to understand atmospheric photooxidation processes involving these acids. Previous studies have postulated that long-chain DCAs can be the precursors of smaller DCAs [9, 10]; the hypothesized mechanisms were subsequently employed by Ervens et al. ${ }^{[11]}$ to show that $\mathrm{C}_{2}-\mathrm{C}_{6}$ DCAs can be formed in cloud droplets through $\mathrm{OH}$ radical oxidation. However, specific oxidation pathways and detail mechanism remain to be explored.

To better understand photooxidation mechanisms of atmospheric DCAs, azelaic acid was selected as the target compound because it can be one of important oxidation products of unsaturated acids, such as oleic acid and linoleic acid ${ }^{[8,12]}$, which can be abundantly present from oceanic origins ${ }^{[13]}$, cooking, traffic and biogenic emissions ${ }^{[14,15]}$. In addition, azelaic acid $\left(\mathrm{C}_{9}\right.$ DCA) has been postulated to be a precursor of smaller atmospheric DCAs ${ }^{[10,16]}$. Experiments were conducted to identify intermediates resulting from photooxidation of azelaic acid in a homogeneous (liquid) reaction system, simulating the reaction environments of cloud or fog droplets. Based on experimental concentration profiles of the intermediates identified in our earlier work, chemometric analyses was conducted in this work to examine the degradation pathway of azelaic acid and the subsequent formation of secondary DCA intermediates.

\section{MATERIALS AND METHODS}

Liquid-phase photooxidation system: Photooxidation of azelaic acid was carried out in a semi-batch fashion in a liquid-phase photooxidation system, consisting of an annular cylindrical reactor with a quartz sleeve at the center of the reactor to house the UV light source (15 W, Ster-L-Ray ${ }^{\mathrm{TM}}$, Atlantic Ultraviolet Corp., USA). The UV light source provided a principal output at 254 $\mathrm{nm}$ and a small amount of radiation at $185 \mathrm{~nm}$, which would induce the formation of ozone from dissolved oxygen followed by the generation of $\mathrm{H}_{2} \mathrm{O}_{2}$. In turn, $\mathrm{H}_{2} \mathrm{O}_{2}$ would undergo photolysis (at $254 \mathrm{~nm}$ ) as one of the $\cdot \mathrm{OH}$ sources. In addition, $185 \mathrm{~nm}$ radiation was

Corresponding Author: Department of Chemical and Biochemical Engineering, University of Western Ontario, London, Ontario, N6A 5B9, Canada 
mostly absorbed by water to subsequently produce $\cdot \mathrm{OH}$. This also minimized the photolysis of other compounds in the reaction system. Our laboratory tests showed that $\mathrm{C}_{2}-\mathrm{C}_{9}$ DCAs have negligible absorbance at $254 \mathrm{~nm}$ and exhibited minimal photolysis.

In the semi-batch reactor, $150 \mathrm{~mL}$ solution (in ultrapure DI water) was circulated at a flow rate of 0.15 $\mathrm{L} \min ^{-1}$, with a residence time outside the reactor for less than half a minute. About $0.5 \mathrm{~mL}$ aliquot was sampled every $45 \mathrm{~min}$; depending on degradation rate at individual reaction conditions; the total volume of the withdrawn aliquot was less than $5 \%$ (by volume) of the circulated solution.

Intermediates identification: Each time $50 \mu \mathrm{L}$ of reactant solution was withdrawn for chemical identification and quantification during the experiment. The aliquot was dried using mild nitrogen, before $50 \mu \mathrm{L}$ of tetrahydrofuran (THF, Merck, Germany) was added to re-dissolve the compounds. About 20-30 min after the addition of $20-\mu \mathrm{L}$ of N,O-bis(trimethylsilyl)trifluoroacetamide (BSTFA, Sigma, USA), a derivatization reagent, $1 \mu \mathrm{L}$ of derivatized sample was immediately injected into a gas chromatograph coupled with a mass spectrum detector (GC-MS, Hewlett Packard 6890, Hewlett Packard 5973, USA) equipped with an HP-5MS capillary column (5\% phenyl methyl siloxane, $30.0 \mathrm{~m} \times 250 \mu \mathrm{m} \times 0.25 \mu \mathrm{m}$, Agilent, USA). The injected sample underwent a temperature program for separation beginning at $60^{\circ} \mathrm{C}$ for 3 min followed by temperature ramping of $8^{\circ} \mathrm{C} / \mathrm{min}$ up to $280^{\circ} \mathrm{C}$ and constant at $280^{\circ} \mathrm{C}$ for $3 \mathrm{~min}$. 1-phenyldodecane (1-PD, Aldrich, USA) was employed as the co-injection standard to monitor the injection loss and instrumental performance. Four repeated analyses of a standard mixture consisting of $\mathrm{C}_{2}-\mathrm{C}_{9}$ DCAs $\left(\mathrm{C}_{2}\right.$ and $\mathrm{C}_{6}$ DCAs, Merck, Germany; $\mathrm{C}_{3}-\mathrm{C}_{5}, \mathrm{C}_{7}$ and $\mathrm{C}_{9}$ DCAs, Aldrich, USA; $\mathrm{C}_{8}$ DCA, Fluka, Switzerland) showed satisfactory recoveries of the tested procedure between $99 \pm 1 \%$ and $103 \pm 2 \%$.

\section{RESULTS AND DISCUSSION}

As illustrated in Fig. 1, azelaic acid decayed exponentially as a first-order reaction, which can be described as,

$\frac{d\left[C_{9-D C A}\right]}{d t}=-k_{1, o b s}\left[C_{9-D C A}\right]$

Where $t$ is the reaction time; $\left[C_{9-D C A}\right]$ represents the concentration of azelaic acid and $k_{1, o b s}$ is the observed first-order rate constant. Although, a number of active

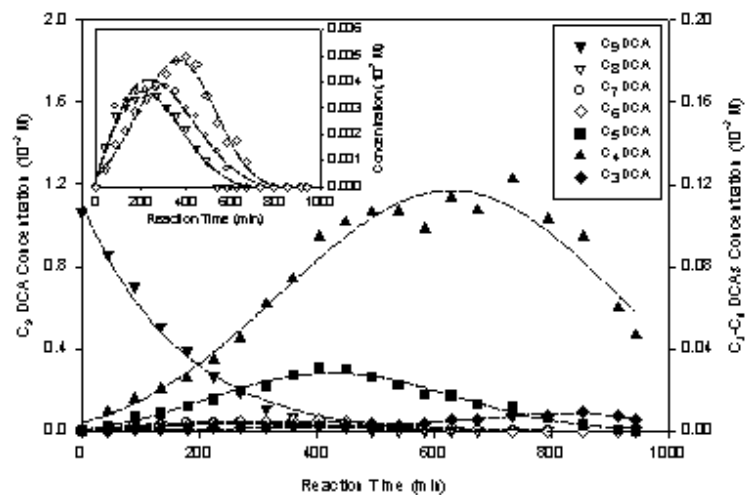

Fig. 1:Concentration profiles of secondary $\mathrm{C}_{3}-\mathrm{C}_{9}$ dicarboxylic acids during the photooxidation of azelaic acid. The inset shows the enlarged concentration profiles of the secondary $\mathrm{C}_{6}-\mathrm{C}_{8}$ dicarboxylic acids

Table 1:The second-order rate constants of $\mathrm{C}_{4}-\mathrm{C}_{9}$ DCAs with hydroxyl radical

\begin{tabular}{ll}
\hline DCA & Rate Constant $\left(\mathrm{M}^{-1} \mathrm{~s}^{-1}\right)$ \\
\hline $\mathrm{C}_{9}$ DCA & $5.4 \times 10^{9}$ \\
$\mathrm{C}_{8}$ DCA & $4.8 \times 10^{9}$ \\
$\mathrm{C}_{7}$ DCA & $3.5 \times 10^{9}$ \\
$\mathrm{C}_{6}$ DCA & $2.0 \times 10^{9}$ \\
$\mathrm{C}_{5}$ DCA & $8.3 \times 10^{8}$ \\
$\mathrm{C}_{4}$ DCA & $3.1 \times 10^{8}$ \\
${ }^{\mathrm{a}}{ }^{\mathrm{E}}$ Except for $\mathrm{C}_{4}$ DCA (from Cabelli et al. $^{[18]}$ ), all listed rate constants \\
are taken from Scholes and Willson ${ }^{[19]}$
\end{tabular}

species are formed along with $\cdot \mathrm{OH}$ radical, effects of $\mathrm{HO}_{2}$ and $\mathrm{O}_{3}$, on azelaic acid degradation are negligible [17] and $\cdot \mathrm{OH}$ is the key oxidant in the reaction system. Thus, the degradation rate of azelaic acid can be expressed as,

$\frac{d\left[C_{9-D C A}\right]}{d t}=k_{2, C_{9-D C A}}[\cdot O H]\left[C_{9-D C A}\right]$

Here, $k_{2, C_{9-D C A}}$ is the second-order rate constant of azelaic acid with $\cdot \mathrm{OH}$ and $[\cdot \mathrm{OH}]$ is the concentration of $\cdot \mathrm{OH}$ radical. However, the apparent observed firstorder degradation kinetics of azelaic acid indicates that $\cdot \mathrm{OH}$ concentration is at steady state during the decomposition of azelaic acid. Hence, comparing Equation (1) and Equation (2), the steady state concentration of $\cdot \mathrm{OH}$ can be estimated based on the following relationship,

$k_{1, o b s}=k_{2, C_{9-D C A}} \cdot[\mathrm{OH}]_{s s}$

Where $[\cdot \mathrm{OH}]_{\mathrm{ss}}$ is the steady state concentration of $\cdot \mathrm{OH}$.

All the identified secondary DCAs had a bell-shape concentration profile (shown in Fig. 1) with a maximum concentration $\left(c_{\max }\right)$ at corresponding time of $t_{\max }$. Succinic acid $\left(\mathrm{C}_{4} \mathrm{DCA}\right)$ and glutaric acid $\left(\mathrm{C}_{5} \mathrm{DCA}\right)$ 
Am. J. Environ. Sci., 4 (5): 462-466, 2008

Table 2: The calculated stoichiometric coefficients of secondary DCAs and corresponding correlation coefficient $\left(r^{2}\right)$ during the photoninduced $\cdot \mathrm{OH}$ oxidation of $\mathrm{C}_{9}-\mathrm{C}_{7}$ DCA

\begin{tabular}{|c|c|c|c|c|c|c|}
\hline Initial DCA & \multicolumn{6}{|c|}{ Stoichiometric Coefficients of Secondary DCA $\left(r^{2}\right)$} \\
\hline & $\mathrm{C}_{9} \mathrm{DCA}$ & $\mathrm{C}_{8} \mathrm{DCA}$ & $\mathrm{C}_{7} \mathrm{DCA}$ & $\mathrm{C}_{6} \mathrm{DCA}$ & $\mathrm{C}_{5} \mathrm{DCA}$ & $\mathrm{C}_{4} \mathrm{DCA}$ \\
\hline $\mathrm{C}_{9}$ DCA & - $(0.99)$ & $7.9 \times 10^{-3}(0.99)$ & $7.8 \times 10^{-3}(0.98)$ & $4.6 \times 10^{-3}(0.95)$ & $1.7 \times 10^{-2}(0.93)$ & $3.8 \times 10^{-2}(1.00)$ \\
\hline $\mathrm{C}_{8}$ DCA & - & $-(0.99)$ & $2.0 \times 10^{-2}(0.94)$ & $2.1 \times 10^{-2}(0.92)$ & $4.7 \times 10^{-2}(0.85)$ & $0.11(0.87)$ \\
\hline $\mathrm{C}_{7} \mathrm{DCA}$ & - & - & $-(0.98)$ & $3.5 \times 10^{-2}(0.84)$ & $5.5 \times 10^{-2}(0.83)$ & $0.31(0.89)$ \\
\hline
\end{tabular}

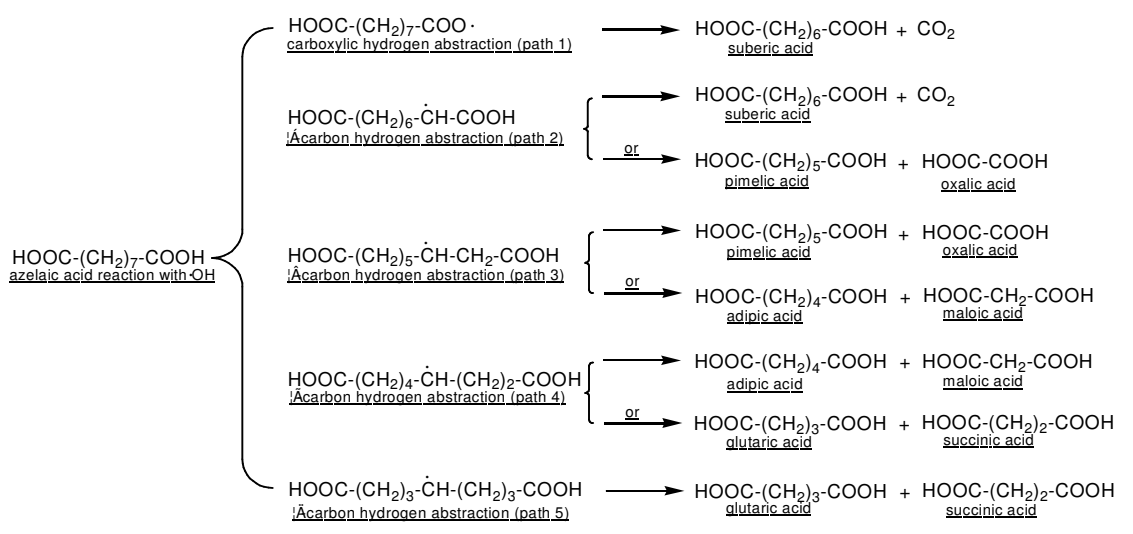

Fig. 2:Proposed reaction pathway for identified secondary dicarboxylic acids during photooxidation of azelaic acid

were most abundant among the secondary $\mathrm{C}_{3}-\mathrm{C}_{8}$ DCAs. $\mathrm{C}_{2}$ DCA (oxalic acid) was not identified during the degradation of azelaic acid, which is due to its high degradation rate observed in our experiments conducted with only oxalic acid (not shown here). The formation of $\mathrm{C}_{8}$ DCA (suberic acid) supports the postulation of Kawamura and Sakaguchi ${ }^{[10]}$ that larger DCAs can undergo sequential decarboxylation to form smaller DCAs. Figure 2 presents the potential formation pathway of secondary dicarboxylic acids, where photooxidation of azelaic acid can be initiated through $\mathrm{H}$-abstraction by $\cdot \mathrm{OH}$ at 5 different locations (paths $1-5$, Fig. 2), forming corresponding DCA radicals prior to further oxidation to generate secondary DCAs. The formation pathway of secondary DCAs from $\cdot \mathrm{OH}$ oxidation of the parent azelaic acid can be qualitatively expressed as,

$$
\begin{aligned}
& C_{9-D C A}+O H \rightarrow \gamma_{C_{8-D C A}} \cdot C_{8-D C A}+\gamma_{C_{7-D C A}} \\
& \cdot C_{7-D C A}+\gamma_{C_{6-D C A}} \cdot C_{6-D C A}+\gamma_{C_{5-D C A}} \cdot C_{5-D C A} \\
& +\gamma_{C_{4-D C A}} \cdot C_{4-D C A}+\gamma_{C_{3-D C A}} \cdot C_{3-D C A}+\text { others }
\end{aligned}
$$

Here $\gamma_{C_{n-D C A}}$ is stoichiometric coefficient of individual secondary DCAs $\left(\mathrm{C}_{\mathrm{n}}\right.$ DCA). To determine the corresponding stoichiometric coefficients $\gamma_{C_{n-D C A}}$ in Equation (4), following equations are established using simple chemometric approach:

$$
\begin{aligned}
& \frac{d\left[C_{8-D C A}\right]}{d t}=\gamma_{C_{8-D C A}} k_{2, C_{9-D C A}}[\cdot O H]_{s s}\left[C_{9-D C A}\right]-k_{2, C_{8-D C A}}[\cdot O H]_{s s}\left[C_{8-D C A}\right](5) \\
& \frac{d\left[C_{7-D C A}\right]}{d t}=\gamma_{C_{7-D C A}} k_{2, C_{9-D C A}}[\cdot O H]_{s s}\left[C_{9-D C A}\right]-k_{2, C_{7-D C A}}[\cdot O H]_{s s}\left[C_{7-D C A}\right](6) \\
& \frac{d\left[C_{6-D C A}\right]}{d t}=\gamma_{C_{6-D C A}} k_{2, C_{9-D C A}}[\cdot O H]_{s s}\left[C_{9-D C A}\right]-k_{2, C_{6-D C A}}[\cdot O H]_{s s}\left[C_{6-D C A}\right](7) \\
& \frac{d\left[C_{5-D C A}\right]}{d t}=\gamma_{C_{s-D C A}} k_{2, C_{9-D C A}}[\cdot O H]_{s s}\left[C_{9-D C A}\right]-k_{2, C_{-D C A}}[\cdot O H]_{s s}\left[C_{5-D C A}\right](8) \\
& \frac{d\left[C_{4-D C A}\right]}{d t}=\gamma_{C_{4-D C A}} k_{2, C_{9-D C A}}[\cdot O H]_{s s}\left[C_{9-D C A}\right]-k_{2, C_{4-D C A}}[\cdot O H]_{s s}\left[C_{4-D C A}\right]
\end{aligned}
$$

Where $k_{2, C_{n-D C A}}$ is the second-order rate constant of corresponding $\mathrm{C}_{\mathrm{n}}$ DCA reaction with $\cdot \mathrm{OH}$; and $\left[C_{n-D C A}\right]$ is the concentration of corresponding secondary $C_{n}$ DCA. It should be noted that, other potential intermediates may subsequently proceed with additional reactions to contribute to the corresponding DCAs in Equations (5-9). However, it can be seen from Fig. 1 that the maximum concentrations of $\mathrm{C}_{8}$ and $\mathrm{C}_{7}$ DCAs occur around $180 \mathrm{~min}$ whereas most identified intermediates such as 4-hydroxy-butanoic acid (one of potential $\mathrm{C}_{4}$ DCA precursors, $t_{\max }=315 \mathrm{~min}$ ) showed a $t_{\max }$ later than $180 \mathrm{~min}$ and by this time $60 \%$ azelaic acid has degraded. Thus the corresponding contribution to DCAs from these intermediates can be neglected in this period when formation of these intermediates dominates over their degradation in the reaction system. In addition, here $\mathrm{C}_{3}$ DCA (malonic acid) were not included in the calculation because in addition to $\cdot \mathrm{OH}$ 
oxidation, this compound can undergo self-degradation through a six-centered cyclic transition state ${ }^{[17]}$; the corresponding experimentally obtained concentrations are excluded in this modeling.

Based on the available secondary order rate constant, $k_{2, C_{n-D C A}}$, of DCAs shown in Table 1 , we solve ordinary differential equations (ODEs) (Equations (1-3) along with Equations (5-9)) using MATLAB and summarize the solved stoichiometric coefficients in Table 2. So the above Equation (4) can be rewritten as,

$$
\begin{aligned}
& C_{9-D C A}+\cdot \mathrm{OH} \rightarrow 7.9 \times 10^{-3} \cdot C_{8-D C A}+7.8 \times 10^{-3} . \\
& C_{7-D C A}+4.6 \times 10^{-3} \cdot C_{6-D C A}+1.7 \times 10^{-2} \cdot C_{5-D C A} \\
& +3.8 \times 10^{-2} \cdot C_{4-D C A}+\text { others }
\end{aligned}
$$

Likewise, the experiments were also conducted using individual $\mathrm{C}_{8}$ DCA (suberic acid) and $\mathrm{C}_{7}$ DCA (pimelic acid) as initial reactants (Fig. 3 and 4). Following the same approach of formulating secondary DCAs resulting from azelaic acid oxidation with $\cdot \mathrm{OH}$ shown above, stoichiometric equations of $\mathrm{C}_{8}$ and $\mathrm{C}_{7}$ DCAs are established as,

$$
\begin{aligned}
& C_{8-D C A}+\cdot O H \rightarrow 2.0 \times 10^{-2} \cdot C_{7-D C A} \\
& +0.11 \cdot C_{4-D C A}+\text { others } \\
& C_{7-D C A}+\cdot O H \rightarrow 3.5 \times 10^{-2} \cdot C_{6-D C A}+5.5 \times 10^{-2} \\
& \cdot C_{5-D C A}+0.31 \cdot C_{4-D C A}+\text { others }
\end{aligned}
$$

Table 2 shows that the above calculated stoichiometric coefficients of Equations (10-12) for $\mathrm{C}_{4}$ and $\mathrm{C}_{5}$ DCAs are much higher than those of other secondary DCAs during the photooxidation of individual $\mathrm{C}_{7}-\mathrm{C}_{9}$ DCAs, suggesting that the sequential decarboxylation suggested by Kawamura and Sakaguchi ${ }^{[10]}$ can not be the dominant reaction pathway. A more direct decomposition, such as breakage of the center C-C bonds via $\mathrm{OH}$-hydrogen abstraction of carbon located at the middle position of parent $\mathrm{C}_{7}-\mathrm{C}_{9}$ DCAs (e.g., paths 4 and 5 for oxidation of $\mathrm{C}_{9}$ DCA shown in Fig. 2) could substantially contribute to smaller DCAs. This observation can be indirectly supported by theoretical and experimental estimation of Serpone et al. ${ }^{[20]}$, who showed that non- $\alpha$-positioned carbons of $\mathrm{C}_{4}$ and $\mathrm{C}_{5}$ monocarboxylic acids (MCAs) have a higher density of frontier electrons and thus are preferably attacked by $\cdot \mathrm{OH}$. Using electron paramagnetic resonance (EPR), Dixon et al. [21] observed free radical intermediates of aliphatic carboxylic acid photodegradation (e.g. acetic acid, propanoic acid), also suggested that $\mathrm{H}$ abstraction occurs preferentially at carbon atom with the longest distance away from the carboxylic group.

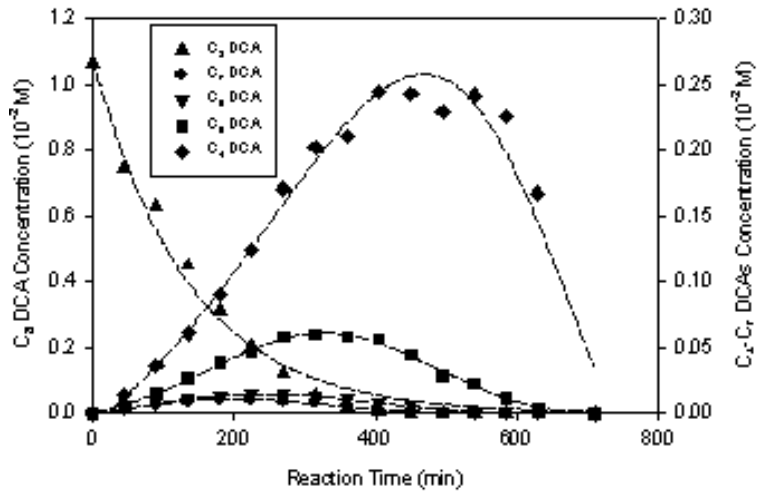

Fig. 3: Concentration profiles of secondary $\mathrm{C}_{4}-\mathrm{C}_{7}$ dicarboxylic acids during the photooxidation of $\mathrm{C}_{8}$ dicarboxylic (suberic) acid

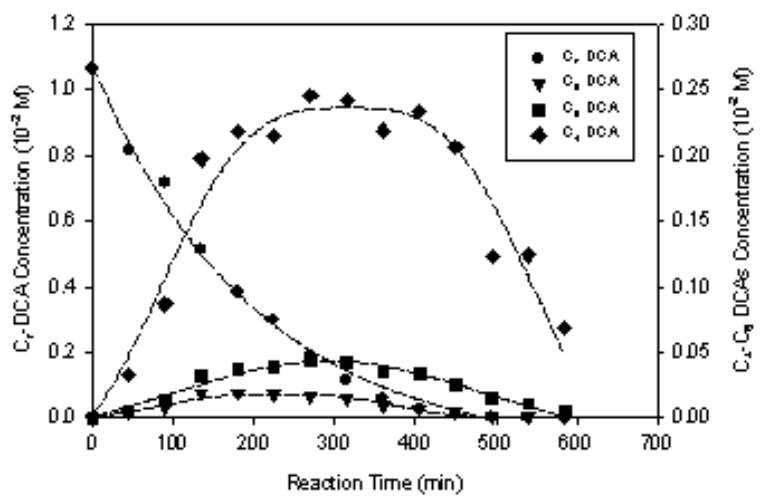

Fig 4: Concentration profiles of secondary $\mathrm{C}_{4}-\mathrm{C}_{6}$ dicarboxylic acids during the photooxidation of $\mathrm{C}_{7}$ dicarboxylic (pimelic) acid

Consistently, Taniguchi et al. ${ }^{[22]}$ found that the abstraction of $\mathrm{H}$ atom of monocarboxylic acid (MCA) by $\cdot \mathrm{OH}$ more actively occurred at $\mathrm{C}-\mathrm{H}$ bond of the methyl groups than at those adjacent to the carboxylic groups. This could be explained by electron density at individual locations of the MCA molecules; carboxylic groups tend to draw electron from immediate adjacent methyl groups, resulting methyl groups away from the carboxylic group become relatively richer in electron density. Hence, $\cdot \mathrm{OH}$, which is electrophilic, would preferably abstract $\mathrm{H}$ from methyl groups farther away from carboxylic groups. However, the above reaction mechanism has been proposed based on simple experiments, fundamental quantum calculation and 
experimental identification of the reactive radicals are needed to verify abovementioned reaction mechanisms of carboxylic acids.

\section{CONCLUSION}

Secondary DCAs identified during photooxidation of individual $\mathrm{C}_{9}-\mathrm{C}_{7}$ DCAs in a liquid-phase system confirmed the hypothesis that longer DCAs can be the precursors of shorter DCAs. Based on chemometric analyses using experimental data, the stoichiometric coefficients for $\mathrm{C}_{4}$ and $\mathrm{C}_{5}$ DCAs are determined, which are much larger than those of other secondary DCAs, suggesting that sequential decarboxylation mentioned in literature is unlikely the dominant pathway; other reaction routes such as preferential abstraction of hydrogen by $\cdot \mathrm{OH}$ from the central carbon of DCA molecules could play an import role. Fundamental quantum calculations and experimental identification of radicals are needed to verify the reaction mechanisms of DCAs derived based on resultant intermediates.

\section{REFERENCES}

1. Satsumabayashi, H., H. Kurita, Y. Yokouchi and H. Ueda, 1989. Mono- and dicarboxylic acids under long-range transport of air pollution in central Japan. Tullus, 41B: 219-229.

2. Jacobson, M.Z., 2001. Global direct radiative forcing due to multicomponent anthropogenic and natural aerosols. J. Geophys. Res., 106:1551-1568.

3. Jacobson, M.Z., 2001. Strong radiative heating due to the mixing state of black carbon in atmospheric aerosols. Nature, 409: 695-697.

4. Lohmann, U. and J. Feichter, 2005. Global indirect aerosol effects: A review. Atmos. Chem. Phys., 5: 715-737.

5. Chung, S.H. and J.H. Seinfeld, 2002. Global distribution and climate forcing of carbonaceous aerosols. J. Geophys. Res., pp: 107: 4407.

6. Edney, E.O., D.J. Driscoll, R.E. Speer, W.S. Weathers, T.E. Kleindienst, W. Li and D.F. Smith, 2000. Impact of aerosol liquid water on secondary organic aerosol yields of irradiated Toluene/propylene/ $\mathrm{NO}_{x} /\left(\mathrm{NH}_{4}\right)_{2} \mathrm{SO}_{4} /$ air mixture. Atmos. Environ., 34: 3907-3919.

7. Kalberer, M., J. Yu, R.G. Cocker, R.C. Flagan, J.H. Seinfeld, 2000. Aerosol formation in the cyclohexeneozone system. Environ. Sci. Technol., 34: 4894-4901.

8. Moise, T. and Y. Rudich, 2002. Reactive uptake of ozone by aerosol-associated unsaturated fatty acids: kinetics, mechanism and products. J. Phys. Chem. A., 106: 6469-6476.

9. Kawamura, K. and K. Ikushima, 1993. Seasonal changes in the distribution of dicarboxylic acids in the urban atmosphere. Environ. Sci. Technol., 27: 22272235.
10. Kawamura, K. and F. Sakaguchi, 1999. Molecular distributions of water soluble dicarboxylic acids in marine aerosols over the Pacific Ocean including tropics. J. Geophys. Res., 104: 3501-3509.

11. Ervens, B., G. Feingold, G.J. Frost and S.M. Kreidenweis, 2004. A modeling study of aqueous production of dicarboxylic acids: 1. Chemical pathways and speciated organic mass production. J. Geophys. Res., 109: D15205, 4440.

12. Katrib, Y., S.T. Martin, H.M. Hung, Y. Rudich, H. Zhang, J.G. Slowik, P. Davidovits, J.T. Jayne and D.R. Worsnop, 2004. Products and mechanisms of ozone reactions with oleic acid for aerosol particles having core-shell morphologies. J. Phys. Chem. A, 108: 66866695.

13. Barger, W.R. and W.D. Garrett, 1970. Surface active organic material in the marine atmosphere. J. Geophys. Res., 75: 4561-4566.

14. Rogge, W.F., L.M. Hildemann, M.A. Mazurek, G.R. Cass and B.R.T. Simoneit, 1993. Sources of fine organic aerosol. 4. Particulate abrasion products from leaf surfaces of urban plants. Environ. Sci. Technol., 27: 2700-2711.

15. Limbeck, A. and H. Puxbaum, 1999. Organic acids in continental background aerosols. Atmos. Environ., 33: 1847-1852.

16. Yu, L.E., M.L. Shulman, R. Kopperud and L.M. Hildemann, 2005. Characterization of organic compounds collected during southeastern aerosol and visibility study: water-soluble organic species. Environ. Sci. Technol., 39: 707-715.

17. Nepotchatykh, O.V. and P.A. Ariya, 2002. Degradation of dicarboxylic acids $\left(\mathrm{C}_{2}-\mathrm{C}_{9}\right)$ upon liquid-phase reactions with $\mathrm{O}_{3}$ and its atmospheric implications. Environ. Sci. Technol., 36: 3265-3269.

18. Cabelli, D.E. and B.H.J. Bielski, 1985. A pulse radiolysis study of some dicarboxylic acids of the citric acid cycle. The kinetics and spectral properties of the free radicals formed by reaction with the $\mathrm{OH}$ radical. Z. Naturforsch. B, Anorg. Chem., Org. Chem., 40B: 1731-1737.

19. Scholes, G. and R.L. Willson, 1967. $\gamma$ - Radiolysis of aqueous thymine solutions. Determination of relative reaction rates of $\mathrm{OH}$ radicals. Trans. Faraday Soc., 63: 2983-2993.

20. Serpone, N., J. Martin, S. Horikoshi and H. Hidaka, 2005. Photocatalyzed oxidation and mineralization of C1-C5 linear aliphatic acids in UV-irradiated aqueous titania dispersions - kinetics, identitification of intermediates and quantum yields. J. Photochem. Photobiol. A: Chem., 169: 235-251.

21. Dixon, W.T., R.O.C. Norman and A.L. Buley, 1964. Electron spin resonance studies of oxidation. Part II. Aliphatic acids and substituted acids. J. Chem. Soc., pp: 3625-3634.

22. Taniguchi, H., K. Fukui, S.I. Ohnishi, H. Hatano, H. Hasegawa and T. Maruyama, 1968. Free-radical intermediates in the reaction of the hydroxyl radical with amino acids. J. Phys. Chem. 72: 1926-1931. 